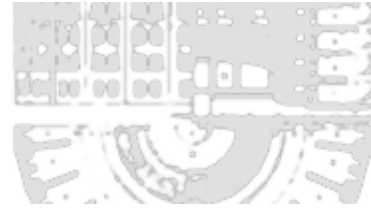 \\ Surveillance \& Society \begin{tabular}{l|l} 
Debate & $\begin{array}{l}\text { The Body-worn Camera as a Transitional } \\
\text { Technology }\end{array}$
\end{tabular}
}

\section{Tjerk Timan}

Tilburg Institute for Law, Technology and Society, Tilburg University, The Netherlands. t.timan@uvt.nl

\section{The Body-worn Camera as Experiment}

In 2009, body-worn cameras were introduced in the Netherlands as an experiment to reduce violence against public servants, including police officers. ${ }^{1}$ Following the allegedly positive results of body-worn cameras in reducing violence in the United Kingdom, ${ }^{2}$ these technologies were introduced with high expectations by several Dutch police forces. However, questions remain about the goals and purposes of body-worn camera adoption. Can a camera prevent or reduce violence against its carrier/wearer? Is it yet another tool to surveil citizens, packaged in a discourse of worker safety? Do the cameras alter police practices and, if so, how? Based on fieldwork conducted in 2010 and 2011 in the Netherlands, this contribution to the debate explores the goals and necessity of this equipment. Questioning the police body camera in this way touches upon a larger question within Surveillance Studies, which is how to look at new tools of surveillance and to what extent they alter surveillance theories, concepts, and practices. Focusing on the new and visible risks over-analyzing and over-emphasizing the tool-in-itself, thereby possibly missing the organizational structure in which the new tool will operate (see Bowker and Starr 1999). Moreover, the body-worn camera in current form is a transitional technology; it will probably be phased out and mutate into another wearable camera technology. ${ }^{3}$

\section{The Need for Body-worn Cameras in the Public Workplace}

To understand the body-worn camera's introduction, it is necessary to determine who is championing it. In the Netherlands, policymakers and politicians framed the body-worn camera within the context of violence against public servants, specifically against train and tram conductors, ambulance personnel, and police officers. It was claimed equipping public servants with body-worn cameras would reduce this violence. Using rhetoric similar to defending CCTV cameras in public space (Brands et al. 2015), the argument goes that the camera's presence has a preventive effect: potential wrong-doers recognize the camera and, having internalized potential consequences of their behavior being recorded, would refrain from doing harm. The idea is that, in these instances, the body-worn camera works as both a warning sign and a potential objective witness during encounters between public servants and citizens. This would make the workplace, which for public servants is often part of public space, safer for those working in it.

\footnotetext{
${ }^{1}$ See for instance http://www.bbc.com/news/uk-england-london-26810753 (accessed March 16th, 2016).

${ }^{2}$ See for instance http://www.bbc.com/news/uk-england-london-27313500 and Woolf (2015). In the UK, body cameras will become part of standard police equipment.

${ }^{3}$ Such as AR-glasses, see Daniels (2016).
} 
However, it remains to be seen whether the body-worn camera can fulfill all these complex goals. That outcome is questionable because CCTV has been introduced with similar, almost unachievable, goals and, so far, it is difficult to prove its efficacy in reducing crime (see Brands et al. 2015; Armitage 2002). The need for cameras in the workplace in the Netherlands is articulated in the framework of safeguarding police work, which differs significantly from the pro-body-worn camera arguments heard recently in the United States, where the focus is on police transparency and accountability.

The reasons for rolling out body-worn cameras in the Netherlands vary. Besides the dominant camera-asa-tool-for-prevention rhetoric, they should also protect officers by serving as an objective witness. In situations involving legal disputes, doubt about police officer or citizen conduct, or public outcry over a police intervention, body-worn camera footage can act as unfettered, time-stamped proof of what happened not only during the intervention itself, but also immediately preceding an intervention. The latter can counter mobile phone recordings and forms of citizen surveillance (or policing the police) that are sometimes displayed on social media. The argument made by policymakers in favor of body camera introduction is that citizen-made recordings often tell only a partial story and/or could have been tampered with - they shape the image of the police, yet often omit the circumstances that lead to the intervention. Body camera footage can counter and improve this image by showing what really happened. Where the first reason of protection is linked to physical safety of the officer, the second is linked to the image of the police and justifying police interventions. In the Netherlands, body-worn camera footage has not yet been subject to high levels of public visibility, as has occurred elsewhere.

\section{Challenges for Body-worn Camera Acceptance}

There are several challenges to the broad adoption of body-worn cameras in the workplace that must be addressed before they can become part of standard police practice. Body-worn cameras have to compete with other existing technologies within local assemblages (Haggerty and Ericson 2000), meaning the body-worn camera will enter a police workspace already filled with other tools and equipment. Bodyworn cameras will enter into different local routines, processes, and protocols, and each locale has its own set of actors involved in body-worn camera adoption (see Timan and Oudshoorn 2012; Timan and Albrechtslund 2015). Besides the governmental or organizational surveillance infrastructure (such as CCTV and its warning signs, public lighting, sound cameras, walkie-talkies, and police vehicles), the body-worn camera encounters competition from the citizen-carried smartphone-a much lighter device equipped with cameras. In that sense, the body-worn camera resonates with the introduction of smartphones: both are mobile recording devices that increase surveillance-participation (Albrechtslund and Lauritsen 2013) in public space.

\section{Internal Acceptance}

Zooming in, body-worn camera acceptance can be analyzed from two sides: internal — or institutionalacceptance, and external-or social-acceptance. The first deals with questions of organizational adoption, work practice, functional use, and added value in police work. The second deals more with the image of policing and the effects of placing a one-sided and allegedly objective technological witness between officers and citizens. The subject of privacy runs through both, and deals with both the act ofand responses to-recording in public space, as well as the role of recordings made while policing with a camera. On the organizational side, the body-worn cameras observed during fieldwork were not unequivocally and enthusiastically welcomed. Resistance revolves around sentiments of policing the police, distrust amongst colleagues, camera ergonomics, and footage processing, and access (see Schneider 2015; Tyler 2004). In relation to privacy, issues include that of workplace privacy, privacy amongst colleagues on duty, and an increase in police accountability. While undoubtedly police accountability and transparency are positive aims to strive for, having a team of colleagues constantly looking over one's shoulder can have a chilling effect on one's work. In current body-worn camera 
scenarios, the CCTV operating room will also have live-stream access to body-worn camera footage, making it possible to aid and direct the officer on the ground. This potentially diminishes the autonomy of the officer(s) in making judgments or 'reading' the situation. Where these judgment calls can be augmented with more 'objective' calls from the operating room, during observations, internal resistance to body-worn camera use was often related to a diminishing appreciation for 'human police work' and an apparent lack of trust in police officers' judgments.

There were mixed results amongst police officers who have varying levels of enthusiasm about wearing a body-worn camera. The ergonomic complaints offered by police officers I interviewed were almost uniform. The clunky interface box and rather difficult setup of clips and cables makes it not only a hassle to use, but also potentially dangerous. Police officers interviewed mentioned that the camera distracts from the job at hand and that the connected cable between the interface box and the actual camera could be used as a weapon against them (as a strangling chord) - thereby actually increasing violence against police, rather than diminishing it. During my fieldwork, most of the cameras were also non-functioning due to a lack of maintenance and preparation, such as cleaning out the memory card before the next shift. In prioritizing technical tools used while on duty, the camera was more often referred to as being a burden rather than an advantage. These problems are also compounded by limited possibilities for the police officer to interact with the camera device once on duty, and reviewing and tagging footage rarely happened because it simply took too much time and effort to accomplish.

\section{External Acceptance}

During observations, which took place in the evenings and during late night shifts in different cities (Rotterdam and Enschede) in the Netherlands, citizens were rarely notified that a body-worn camera was in place or was recording them. This practice is at odds with the initial goal established during the introduction of the camera in the Netherlands; namely, to have the camera diminish violence against police officers by acting as a preventive tool. The body-worn camera worn in public space is not very visible. It is a dark, small, black object that comes in different shapes and sizes: the cameras studied consisted of a small tube-camera and a set of clips. There was also a clip-on camera that resembles a microphone. The camera can be carried in various ways (via clips the camera can be mounted on a helmet, shoulder, or breast-pocket). The camera can be controlled via a wrist-band, making the camera hands-free. This influences what a camera is - not only for the camera's carrier, but also for those being potentially watched. The act of video recording is also disconnected from the archetypical physical set of actions normally related to camera use (be it a classical camera or a smartphone) - in the current body-worn camera setup, aiming and recording are no longer recognizable. This allows for obfuscated or secret recording of citizens by the body camera-carrier - not only are citizens not made aware of the body-worn camera's presence, but they may also hardly recognize it as a camera.

A primary threat to privacy thus lies at the intersection between the (lack of) visibility of the actual camera and the possibility for officers to make covert recordings in public space. A second and related threat to privacy lies in the identifiability of the watched. The point-of-view of the body-worn camera differs drastically from CCTV cameras, because it is worn on body of its carrier. The footage from body-worn cameras provides a different 'gaze' upon the watched, making the recording more intimate - as the camera is mainly used once the agent has started to engage with a citizen, in close proximity of the subject of recording. The step from current practice to the integration of automated face recognition is not hard to imagine, especially since body-worn cameras have started to stream their data. 


\section{Dealing with Body Camera Data and Privacy}

Besides the act of recording in public space that creates multiple privacy threats, a second stage in bodyworn camera use is the processing of footage. There are multiple scenarios of data use here, which have different legal consequences for privacy, but also have different implications for privacy expectations and/or experiences. In the context of my fieldwork, once footage was taken in public space, the responsible agent could not thereafter tamper with the footage (by deleting or editing); only the tagging of footage was allowed. Back at the station, a dedicated body-worn camera-officer was in charge of transferring the stored footage onto a local computer. As a matter of security protocol, this computer was not to be connected to any network: it remained stand-alone and not remotely accessible. Only after the compulsory reports had been written could the agent who carried the body-worn camera review the (tagged) footage to decide in conjunction with the responsible body-worn camera-officer(s) what to save and what to delete. In this scenario, potential personal data of the watched seem well-protected and safeguards and protocols seem to be in place to make sure footage is only used for specific purposes. However, these purposes are not clearly set or communicated, neither to the watched nor to local police personnel. In the cases observed, this resulted in stills from footage being used the next day or evening in briefings, and in data being shared across multiple computers and internal police networks, thereby ignoring data safeguard practices.

The privacy implications here can be found in the mismatch between legal provisions and principles (purpose limitation or anonymization) and local infrastructures and practices; where the new privacy implications stem from the type of data body-worn cameras produce. As noted above, body-worn cameras document more sensitive personal information due to their angle of recording: faces and more detailed features are being recording at eye/shoulder/helmet-height. One could argue this makes surveillance fairer because as a subject one can adjust and more freely 'dodge' surveillance (for instance by turning around and walking away). However, in practice, the body-worn camera is hardly recognized as an archetypical camera by subjects. As a result, it is being used to secretly record citizens. From a larger context, however, the body-worn camera as a new camera in public space does not particularly stand out or 'impress' - most subjects interviewed were not shocked by the presence of the camera, pointing out they carried one themselves. Rather, most worries were geared towards data processing. Compared to the (almost) ubiquitous presence of smartphones, the body-worn camera comes across as an asynchronous and clunky tool with limited image quality and battery time. In that sense, the main privacy threats are found more downstream in data management practices than in the original recording.

\section{Conclusion}

The future of body-worn cameras in the Netherlands is unclear. What we can learn from their introduction is that, once introduced (when the wow-effect ends) we need to question how the new tool might affect the surveillance landscape over the long term (see Fuchs 2010; Taekke 2011). The current body-worn camera is not likely to stay around, mainly for technological reasons. However, equipping police officers with some form of mobile (and not only) visual recording device is likely to continue. What we can learn from analyzing current body-worn camera technology is how it changes a) the perspective of surveillance (literally more down to earth), b) how it influences power relations between citizens and police (now there is a constant 'objective' witness in between, potentially chilling the interaction between officer and citizen and triggering a camera-race between citizens equipped with smartphones and police equipped with body cameras), and c) how it stretches surveillance over time and space by adding to the pool of surveillancedata in which it becomes more difficult for citizens to be anonymous in the crowd. 


\section{References}

Albrechtslund, Anders, and Peter Lauritsen. 2013. "Spaces of Everyday Surveillance: Unfolding an Analytical Concept of Participation." Geoforum 49: 310-316.

Armitage, Rachel. 2002. To CCTV or not to CCTV: A Review of Current Research into the Effectiveness of CCTV Systems in Reducing Crime. Community safety practice briefing. London: Nacro (Crime and Social Policy Section).

Bowker, Geoffrey and Susan Leigh Starr.1999. Sorting Things Out: Classification and its Consequences. Cambridge, MA: The MIT Press.

Brands, Jelle, Tim Schwanen, and Irina Van Aalst. 2015. "Fear of Crime and Affective Ambiguities in the Night-Time Economy." Urban Studies 52(3): 439-455.

Daniels, Kindel. 2016. "Will augmented reality change policing?” Feb 29, 2016. policeone.com. https://www.policeone.com/police-products/body-cameras/articles/149117006-Will-augmented-reality-change-policing/ (accessed March 16th, 2016).

Fuchs, Christian. 2010. "Web 2.0, Prosumption, and Surveillance.” Surveillance \& Society 8(3): 288-309.

Haggerty, Kevin D., and Richard V. Ericson. 2000. “The Surveillant Assemblage.” The British Journal of Sociology 51(4): 605622.

Schneider, Christopher J. 2015. "Police Image Work in an Era of Social Media: YouTube and the 2007 Montebello Summit Protest." In Social Media, Politics and the State: Protests, Revolutions, Riots, Crime and Policing in an Age of Facebook, Twitter and YouTube, edited by Daniel Trottier and Christian Fuchs, 227-246. London: Routledge.

Taekke, Jesper. 2011. "Digital Panopticism and Organizational Power." Surveillance \& Society 8(4): 441-454.

Timan, Tjerk, and Nelly, E.J. Oudshoorn. 2012. "Mobile Cameras as New Technologies of Surveillance? How Citizens Experience the Use of Mobile Cameras in Public Nightscapes." Surveillance \& Society 10(2): 167-181.

Timan, Tjerk, and Anders Albrechtslund. 2015. "Surveillance, Self and Smartphones: Tracking Practices in the Nightlife." Science \& Engineering Ethics 21: 1-18.

Tyler, Tom R. 2004. "Enhancing Police Legitimacy." ANNALS of the American Academy of Political and Social Science 593(1): 84-99.

Woolf, Christopher. 2015. "UK police: Body cameras make us behave, show world we're not brutes." pri.org. Available at: http://www.pri.org/stories/2015-04-10/us-mulls-police-body-cameras-uk-offers-lessons. pri.org. (accessed March 16th, 2016). 